\title{
Study of Quantile-Based Cumulative Rényi Information Measure
}

\author{
Rekha Rani , Vikas Kumar* \\ Department of Applied Sciences, UIET, M. D. University, Rohtak
}

\begin{abstract}
In this paper, we proposed a quantile version of cumulative Rényi entropy for residual and past lifetimes and study their properties. We also study quantile-based cumulative Rényi entropy for extreme order statistic when random variable untruncated or truncated in nature. Some characterization results are studied using the relationship between proposed information measure and reliability measure. We also examine it in relation to some applied problems such as weighted and equilibrium models.
\end{abstract}

Keywords Rényi entropy, Cumulative residual entropy, Quantile function, Order statistic, Reliability measures, Weighted entropy.

\section{AMS 2010 subject classifications 62N05, 90B25}

DOI: $10.19139 /$ soic-2310-5070-1034

\section{Introduction}

Let $X$ be a random variable with distribution function $F(x)$ and quantile function $Q(u)$. Then, the quantile function of $X$ is defined by

$$
Q(u)=F^{-1}(u)=\inf \{x \mid F(x) \geq u\}, \quad 0 \leq u \leq 1 .
$$

Here and throughout the article, $X$ is absolutely continuous nonnegative random variable with probability density function (pdf) $f(x)$ and survival function $\bar{F}(x)$. If $f($.$) is the pdf of X$, then $f(Q(u))$ and $q(u)=\frac{d Q(u)}{d u}$ respectively known as the density quantile function and the quantile density function (see Parzen (1979)). Using (1.1), we have $F(Q(u))=u$ and differentiating it with respect to $u$ obtain

$$
q(u) f Q(u)=1 .
$$

The mean of the distribution assumed to be finite, is

$$
E(X)=\int_{0}^{1} Q(p) d p=\int_{0}^{1}(1-p) q(p) d p
$$

In certain cases the approach based on quantile functions is more fruitful than the use of cumulative distribution functions, since quantile functions are less influenced by extreme statistical observations. Also, there are certain properties of quantile functions that are not shared by the distribution function approach. The quantile functions used in applied works such as various forms of lambda distributions (van Standen and Loots, 2009), the power-Pareto distribution (Hankin and Lee, 2006), Govindarajulu's distribution do not have tractable distribution functions. For a detailed and recent study on quantile function and its properties in modeling and analysis we refer

*Correspondence to: Vikas Kumar (Email: vikas_iitr82@yahoo.co.in). Department of Applied Sciences, UIET, M. D. University, Rohtak India (124001).

ISSN 2310-5070 (online) ISSN 2311-004X (print)

Copyright (C) 2021 International Academic Press 
to Gilchrist (2000), Nair et al. (2013), Sreelakshmi et al. (2018) and Sharma and Chakrabarty (2017). An important quantile measure useful in reliability analysis is the hazard quantile function, defined as

$$
K(u)=h(Q(u))=\frac{f Q(u)}{(1-u)}=\frac{1}{(1-u) q(u)},
$$

where $h(x)=\frac{f(x)}{1-F(x)}$ is the hazard rate of $X$. Another useful measure closely related to hazard quantile function is the mean residual quantile function, as given by

$$
M(u)=m(Q(u))=(1-u)^{-1} \int_{u}^{1}(1-p) q(p) d p,
$$

where $m(t)=E(X-t \mid X>t)$ is the mean residual life function $(M R L F)$ of $X$. It is well known that both hazard quantile function and mean residual quantile function uniquely determine the quantile density function $q(u)$. Further the relationship between the quantile density function and mean residual quantile function is given by

$$
q(u)=\frac{M(u)-(1-u) M^{\prime}(u)}{(1-u)} .
$$

The idea of the information theoretic entropy was introduced by Shannon (1948) which plays an important role in diverse areas such as financial analysis, data compression, molecular biology, computer science and information theory. The average amount of uncertainty associated with the nonnegative continuous random variable $X$ can be measured using the differential entropy function

$$
H(X)=-\int_{0}^{\infty} f(x) \log f(x) d x
$$

a continuous counterpart of the Shannon (1948) entropy in the discrete case.

Rao et al. (2004) pointed out some basic shortcomings of the Shannon differential entropy measure. Rao et al. (2004) introduced an alternative measure of uncertainty called the cumulative residual entropy (CRE) of a random variable $X$ with survival function $\bar{F}$, given by

$$
\xi(X)=-\int_{0}^{\infty} \bar{F}(x) \log \bar{F}(x) d x .
$$

Asadi and Zohrevand (2007) have considered the dynamic cumulative residual entropy (DCRE) as the cumulative residual entropy of the residual lifetime $X_{t}=[X-t \mid X>t]$. This is given by

$$
\xi(X, t)=-\int_{t}^{\infty} \frac{\bar{F}(x)}{\bar{F}(t)} \log \frac{\bar{F}(x)}{\bar{F}(t)} d x .
$$

Di Crescenzo and Longobardi (2009) introduced a dual measure based on the cumulative distribution function $F(x)$, called the cumulative past entropy (CPE) and its dynamic version as

$$
\begin{gathered}
\bar{\xi}(X)=-\int_{0}^{\infty} F(x) \log F(x) d x, \\
\bar{\xi}(X ; t)=-\int_{0}^{t} \frac{F(x)}{F(t)} \log \frac{F(x)}{F(t)} d x .
\end{gathered}
$$

All the theoretical investigations and applications using these information measures are based on the distribution function. Since a probability distribution can be specified either in terms of distribution function or by the quantile function. Although both convey the same information about the distribution, with different interpretations, the concepts and methodologies based on distribution functions are traditionally employed in most forms of statistical 
theory and practice. The study of entropy functions using quantile functions is of recent interest. Sankaran and Sunoj (2017) have introduced the quantile version of the dynamic cumulative residual entropy (DCRE), which is defined by

$$
\xi(u)=\xi(X ; Q(u))=\frac{\log (1-u)}{(1-u)} \int_{u}^{1}(1-p) q(p) d p-(1-u)^{-1} \int_{u}^{1} \log (1-p)(1-p) q(p) d p .
$$

When $u \longrightarrow 0,(11)$ reduces to $\xi=-\int_{0}^{1}(\log (1-p))(1-p) q(p) d p$, a quantile version of $C R E$. New models and characterizations that are unresolvable in the distribution function approach can be resolved with the help of quantile functions. Quantile functions can be properly employed to formulate properties of entropy function and other information measures for nonnegative absolutely continuous random variables refer to, Sunoj and Sankaran (2012), Sunoj et al. (2013) and Noughabi et al. (2020).

There have been attempts by several authors for the parametric generalization of $C R E$. Zografos and Nadarajah (2005) introduced the cumulative residual Rényi entropy (CRRE) of order $\alpha$ as

$$
\xi^{\alpha}(X)=\frac{1}{(1-\alpha)} \log \left(\int_{0}^{\infty} \bar{F}^{\alpha}(x) d x\right), \alpha>0, \alpha \neq 1 .
$$

Further, Sunoj and Linu (2012) proposed a dynamic version of it as

$$
\xi^{\alpha}(X ; t)=\frac{1}{(1-\alpha)} \log \left(\frac{\int_{t}^{\infty} \bar{F}^{\alpha}(x) d x}{\bar{F}^{\alpha}(t)}\right) .
$$

This define as the dynamic cumulative residual Rényi entropy (DCRRE) of the random variable $X_{t}=[X-t \mid X>t]$. For more properties and applications of this measure, we refer to Kayal (2015) and Minimol (2017).

This measure is much more flexible due to the parameter $\alpha$ enabling several measurements of uncertainty within a given distribution and increase the scope of application. Also this forms a parametric family of entropy measures that give weights to extremely rare and regular events completely different. Some properties and applications of these information theoretic measure in reliability engineering, computer vision, coding Theory and finance have been also studied by several researcher, refer to Rao (2005), Wang and Vemuri (2007) Navarro et al. (2010), Sheraz et al. (2015), Kumar and Singh (2018) and Baratpour and Khammar (2018).

Motivated by these, in the present study we consider survival and distribution function based cumulative residual Rényi entropy (CRRE) (residual and past) entropy measures of order $\alpha$ in terms of quantile functions. The present manuscript we introduce the quantile version of GCRE of order $\alpha$ for residual and reversed residual (past) lifetime and proved some characterization results of these for extreme order statistics.

The text is organized as follows. In Section 2, we introduce the quantile-based cumulative residual Rényi entropy and its dynamic version. Section 3 proves some characterization results based on the measure considered in Section 2. In Section 4, we extend the quantile-based cumulative residual Rényi entropy in the context of order statistics and study its properties. In Section 5, we derive the weighted form of this measure and call it weighted cumulative residual Rényi quantile entropy and study some characterization results.

\section{Cumulative residual Rényi quantile entropy}

The quantile version of cumulative residual Rényi entropy of the nonnegative random variable $X$ corresponding to (12) becomes

$$
\breve{\xi}^{\alpha}=\frac{1}{(1-\alpha)} \log \left(\int_{0}^{1}(1-p)^{\alpha} q(p) d p\right),
$$

and called it cumulative residual Rényi quantile entropy (CRRQE). When $\alpha \rightarrow 1, \breve{\xi}^{\alpha}$ reduces to $-\int_{0}^{1}(\log (1-$ $p)(1-p) q(p) d p$, a quantile version of $C R E$, sugggested by Sankaran and Sunoj (2017). Equation (14) can be 
written as

$$
\breve{\xi}^{\alpha}=\frac{1}{(1-\alpha)} \log \left(\int_{0}^{1} \frac{(1-p)^{\alpha-1}}{K(p)} d p\right) .
$$

Equation (15) is the expressions of $\breve{\xi}^{\alpha}$ in terms of the hazard quantile function $K(u)$ respectively. There are some models that do not have any closed form expressions for cdf or pdf, but have simple quantile function or quantile density functions (see Nair et al. (2011)). Accordingly in the following example, we obtain $\breve{\xi}_{\alpha}$ for which $q($.$) exists.$

\section{Example 1}

Suppose $X$ is distributed with quantile density function given by, $q(u)=(1-u)^{-A}(-\log (1-u))^{-M} ; 0<u<1$ where $\mathrm{M}$ and $\mathrm{A}$ are real constants. Further, it contains several distributions which includes Weibull when $\mathrm{A}=1, \mathrm{M}$ $=\frac{b-1}{b}$, uniform when $\mathrm{A}=0, \mathrm{M}=0$, Pareto when $A>1, \mathrm{M}=0$, and rescaled beta when $A<1, \mathrm{M}=0$. Then the CRRQE is obtained as

$$
\breve{\xi}^{\alpha}=\frac{1}{(1-\alpha)}\{\log \gamma(1-M)-(1-M) \log (1+\alpha-A)\},
$$

where $\gamma($.$) represents the gamma function.$

\section{Example 2}

A lambda family of distribution that is of interest in reliability is the Davis distribution proposed by Hankin and Lee (2006) with quantile function

$$
Q(u)=C u^{\lambda_{1}}(1-u)^{-\lambda_{2}} ; 0<u<1, C, \lambda_{1}, \lambda_{2} \geq 0 .
$$

This is a flexible family for right skewed nonnegative data that provides good approximations to the exponential, gamma, lognormal and weibull distributions. The CRRQE (14), for Davis distribution is given as

$$
\breve{\xi}^{\alpha}=\frac{1}{(1-\alpha)} \log \left\{C \lambda_{1} \beta\left(\lambda_{1}, \alpha-\lambda_{2}+1\right)+C \lambda_{2} \beta\left(\lambda_{1}+1, \alpha-\lambda_{2}\right)\right\}
$$

As $\lambda_{2} \longrightarrow 0,(16)$ reduces to $\breve{\xi}^{\alpha}=\frac{1}{(1-\alpha)} \log \left(C \lambda_{1} \beta\left(\lambda_{1}, 1+\alpha\right)\right)$, corresponding to the Power distribution. Also as $\lambda_{1} \longrightarrow 0,(16)$ reduces to $\breve{\xi}^{\alpha}=\frac{1}{(1-\alpha)} \log \left(\frac{C \lambda_{2}}{\alpha-\lambda_{2}}\right)$, corresponding to the Pareto I distribution.

\section{Example 3}

A nonnegative random variable $X$ is Weibull distributed with quantile function and the quantile density function given by $Q(u)=\left\{\frac{-1}{a} \log (1-u)\right\}^{\frac{1}{b}}, q(u)=\frac{\left\{-\frac{1}{a} \log (1-u)\right\}^{\frac{1}{b}-1}}{a b(1-u)} ; 0<u<1$. Also, the mean of the distribution is $E(X)=\int_{0}^{1} Q(p) d p=\int_{0}^{1}(1-p) q(p) d p$, assumed to be finite then the CRRQE (14) gives

$$
\begin{aligned}
\breve{\xi}^{\alpha} & =\frac{1}{(1-\alpha)} \log \left(\int_{0}^{1}(1-p)^{\alpha} \frac{\left(-\frac{1}{a} \log (1-p)\right)^{\left(\frac{1}{b}-1\right)}}{a b(1-p)} d p\right) \\
& =\frac{1}{1-\alpha} \log \left(\frac{\left(\frac{1}{a}\right)^{\frac{1}{b}-1} \gamma\left(\frac{1}{b}\right)}{a b(\alpha)^{\frac{1}{b}}}\right),
\end{aligned}
$$

and the mean of the distribution is $E(X)=\left(\frac{\left(\frac{1}{a}\right)^{\frac{1}{b}-1} \gamma\left(\frac{1}{b}\right)}{a b}\right)$.Thus, we have $\frac{e^{(1-\alpha) \breve{\xi}^{\alpha}}}{E(X)}=\alpha^{\frac{-1}{b}}$. This result shows that for Weibull family, this ratio is constant. If $b=1$, then $\mathrm{X}$ has exponential distribution and this ratio is equal to $\frac{1}{\alpha}$.

\section{Example 4}

When $X$ is distributed with quantile density function given by $q(u)=K u^{\delta}(1-u)^{-(A+\delta)} ; 0<u<1$ where $\mathrm{K}, \delta$, and $\mathrm{A}$ are real constants. It contains several well known distributions which include the exponential $(\delta=$ 
$0 ; A=1)$, Pareto $(\delta=0 ; A<1)$, rescalded beta $(\delta=0 ; A>1)$, the $\log \operatorname{logestic}$ distribution $(\delta=\lambda-1 ; A=2)$ and Govindarajulu's distribution $(\delta=b-1 ; A=-b, K=a b(b+1))$. Then the cumulative residual Rényi quantile entropy (14) is obtained as

$$
\breve{\xi}^{\alpha}=\frac{1}{(1-\alpha)}\{\log K+\log \beta(1+\delta, 1+\alpha-A-\delta)\} .
$$

In the context of reliability and survival analysis, when the current age of a component need to be taken into account. In such cases, measuring uncertainty using $\breve{\xi}_{\alpha}$ is not appropriate and a modified version of $\breve{\xi}_{\alpha}$ is essential for such residual random variable, $X_{t}=(X-t \mid X>t)$. In this case, dynamic measure is useful to describe the information carried by the random lifetime when age changes. An equivalent definition for the dynamic cumulative residual Rényi entropy (13) in terms of quantile function is given by

$$
\breve{\xi}^{\alpha}(u)=\breve{\xi}^{\alpha}(X ; Q(u))=\frac{1}{(1-\alpha)} \log \left(\frac{1}{(1-u)^{\alpha}} \int_{u}^{1}(1-p)^{\alpha} q(p) d p\right) .
$$

The measure (17) may be considered as the dynamic cumulative residual Rényi quantile entropy (DCRRQE) measure. Rewriting equation (17) and using (6), we come to

$$
e^{(1-\alpha) \breve{\xi}^{\alpha}(u)}=\frac{1}{(1-u)^{\alpha}} \int_{u}^{1}(1-p)^{\alpha-1} M(p) d p-\frac{1}{(1-u)^{\alpha}} \int_{u}^{1}(1-p)^{\alpha} M^{\prime}(p) d p .
$$

Applying integration by parts on the last term and simplifying, we obtain

$$
(1-u)^{\alpha} e^{(1-\alpha) \breve{\xi}^{\alpha}(u)}-M(u)(1-u)^{\alpha}=(1-\alpha) \int_{u}^{1}(1-p)^{\alpha-1} M(p) d p .
$$

Differentiating (18) with respect to $u$ both sides, and using (4), (18) reduces to

$$
q(u)=\left(\frac{\alpha}{1-u}-(1-\alpha) \breve{\xi}^{\prime \alpha}(u)\right) e^{(1-\alpha) \breve{\xi}^{\alpha}(u)},
$$

where prime denote the derivative with respect to $u$. Equation (19) provides a direct relationship between quantile density function $q(u)$ and $\breve{\xi}^{\alpha}(u)$. Therefore $\breve{\xi}^{\alpha}(u)$ uniquely determines the underlying distribution. Table 2.1 provides quantile functions of some important models and corresponding entropies.

where $\bar{\gamma}_{x}(a, b)$ and $\bar{\beta}_{x}(a, b)$ known as the incomplete gamma function and incomplete beta function defined as $\bar{\gamma}_{x}(a, b)=\int_{x}^{\infty} y^{a-1} e^{-b y} d y, a, b>0, x>0$ and $\bar{\beta}_{x}(a, b)=\int_{x}^{1} y^{a-1}(1-y)^{b-1} d x, a, b>0, x>0$ respectively.

The next theorem gives necessary and sufficient conditions for $\breve{\xi}^{\alpha}(u)$ to be an increasing (decreasing) function of $u$.

\section{Theorem 1}

Let $X$ be a nonnegative absolutely continuous random variable having survival function $\bar{F}(x)$, then $\breve{\xi}^{\alpha}(u)$ is increasing (decreasing), if and only if for all $u \in(0,1)$

$$
\breve{\xi}^{\alpha}(u) \geq(\leq) \frac{-\log K(u)-\log \alpha}{1-\alpha} .
$$

Proof

Differentiating (17) both sides with respect to $u$, we obtain

$$
(1-\alpha)(1-u) \breve{\xi}^{\prime}(u)=\alpha-\frac{q(u)(1-u)}{e^{(1-\alpha) \breve{\xi}^{\alpha}(u)}} .
$$

Using (4), we have

$$
(1-\alpha)(1-u) \breve{\xi}^{\prime}(u)=\alpha-\frac{1}{K(u) e^{(1-\alpha) \breve{\xi}^{\alpha}(u)}} .
$$

Since $\breve{\xi}^{\alpha}(u)$ is increasing (decreasing), that is, $\breve{\xi}^{\alpha}(u) \geq(\leq) 0$ and for all $u \in(0,1)$. Hence above expression becomes $\frac{-\log K(u)-\log \alpha}{1-\alpha} \geq(\leq) 0$. This conclude the proof. 
Table 2.1 Mean residual quantile function $M(u)$ and $D C R R Q E \breve{\xi}^{\alpha}(u)$ for various lifetime distributions

\begin{tabular}{|c|c|c|c|}
\hline Distribution & Quantile function $Q(u)$ & $\mathrm{M}(\mathrm{u})$ & $\breve{\xi}^{\alpha}(u)$ \\
\hline Uniform & $a+(b-a) u$ & $\frac{(b-a)(1-u)}{2}$ & $\frac{1}{1-\alpha} \log \left(\frac{(b-a)(1-u)}{\alpha+1}\right)$ \\
Exponential & $-\lambda^{-1} \log (1-u)$ & $\frac{1}{\lambda}$ & $\frac{1}{1-\alpha} \log \left(\frac{1}{\alpha \lambda}\right)$ \\
Gompertz & $\frac{1}{\log c}\left(1-\frac{\log c \log (1-u)}{B}\right)$ & $\frac{1}{B}$ & $\frac{1}{1-\alpha} \log \left(\frac{1}{B \alpha}\right)$ \\
Pareto I & $b(1-u)^{-\frac{1}{a}}$ & $\frac{b(1-u)^{-\frac{1}{a}}}{a-1}$ & $\frac{1}{1-\alpha} \log \left(\frac{b(1-u)^{-\frac{1}{a}}}{a \alpha-1}\right)$ \\
Generalized Pareto & $\frac{b}{a}\left((1-u)^{-\frac{a}{a+1}}-1\right)$ & $b(1-u)^{-\frac{a}{a+1}}$ & $\frac{1}{1-\alpha} \log \left(\frac{b(1-u)^{-\frac{a}{a+1}}}{\alpha a+\alpha-a}\right)$ \\
Finite Range & $b\left(1-(1-u)^{\frac{1}{a}}\right)$ & $\frac{b(1-u)^{\frac{1}{a}}}{a+1}$ & $\frac{1}{1-\alpha} \log \left(\frac{b(1-u)^{\frac{1}{a}}}{a \alpha+1}\right)$ \\
Log logestic & $\frac{1}{a}\left(\frac{u}{1-u}\right)^{\frac{1}{b}}$ & $\frac{\bar{\beta}_{u}\left(\frac{1}{b}, 1-\frac{1}{b}\right)}{a b(1-u)}$ & $\frac{1}{1-\alpha} \log \left(\frac{\bar{\beta}_{u}\left(\frac{1}{b}, \alpha-\frac{1}{b}\right)}{a b(1-u)^{\alpha}}\right)$ \\
Power & $a u^{\frac{1}{b}}$ & $\frac{a \bar{\beta}_{u}\left(\frac{1}{b}, 2\right)}{b(1-u)}$ & $\frac{1}{1-\alpha} \log \left(\frac{a \bar{\beta}^{\left.\frac{1}{b}, \alpha+1\right)}}{b(1-u)^{\alpha}}\right)$ \\
Govindarajulu's & $a\left\{(b+1) u^{b}-b u^{b+1}\right\}$ & $\frac{a b(b+1)}{(1-u)} \bar{\beta}_{u}(b, 3)$ & $\frac{1}{1-\alpha} \log \left(\frac{a b(b+1) \bar{\beta}_{u}(b, \alpha+2)}{(1-u)^{\alpha}}\right)$ \\
Tukey lambda & $\frac{u^{\lambda}-(1-u)^{\lambda}}{\lambda}$ & $\frac{\bar{\beta}_{u}(\lambda, 2)}{(1-u)}+\frac{(1-u)^{\lambda}}{\lambda+1}$ & $\frac{1}{1-\alpha} \log \left(\frac{\bar{\beta}_{u}(\lambda, \alpha+1)}{(1-u)^{\alpha}}+\frac{(1-u)^{\lambda}}{\alpha+\lambda}\right)$ \\
\hline
\end{tabular}

In many realistic situations, the random variable is not necessarily related to future only, but they can also refer to the past. Suppose at time $t$, one has undergone a medical test to check for a certain disease. Let us assume that the test is positive. If we denote by $X$ the age when the patient was infected, then it is known that $X<t$. Now the question is, how much time has elapsed since the patient had been infected by this disease. In this situation, the random variable ${ }_{t} X=[t-X \mid X \leq t]$, which is known as inactivity time is suitable to describe the time elapsed between the failure of a system and the time when it is found to be 'down'.

The past lifetime random variable ${ }_{t} X$ is related with two relevant ageing functions, the reversed hazard rate defined by $\mu_{F}(x)=\frac{f(x)}{F(x)}$, and mean inactivity time defined by $m(t)=E(t-X \mid X<t)=\frac{1}{F(t)} \int_{0}^{t} F(x) d x$. The quantile versions of reversed hazard rate function and mean inactivity time are given as

$$
\bar{K}(u)=\bar{K}(Q(u))=u^{-1} f(Q(u))=[u q(u)]^{-1},
$$

and

$$
\bar{M}(u)=m(Q(u))=u^{-1} \int_{0}^{u}[Q(u)-Q(p)] d p=\frac{1}{u} \int_{0}^{u} p q(p) d p,
$$

respectively. The relationship (6) for inactivity time becomes

$$
q(u)=\frac{\bar{M}(u)+u \bar{M}(u)}{u}
$$

refer to Nair and Sankaran (2009). Analogous to cumulative residual Rényi entropy (CRRE) of order $\alpha$ (12), Abbasnejad (2011) proposed a cumulative entropy measure to the failure entropy and its dynamic version, which are given as

$$
\bar{\xi}^{\alpha}(X)=\frac{1}{(1-\alpha)} \log \left(\int_{0}^{\infty} F^{\alpha}(x) d x\right)
$$

and

$$
\bar{\xi}^{\alpha}(X ; t)=\frac{1}{(1-\alpha)} \log \left(\int_{0}^{t} \frac{F^{\alpha}(x) d x}{F^{\alpha}(t)}\right), t \geq 0
$$


respectively. When $\alpha \longrightarrow 1$, (25) and (24) reduces to cumulative entropy (9) and past cumulative entropy (10) respectively.

Sankaran and Sunoj (2017) have considered the quantile version of cumulative past entropy as

$$
\bar{\xi}(X ; Q(u))=\frac{\log u}{u} \int_{0}^{u} p q(p) d p-u^{-1} \int_{0}^{u} p(\log p) q(p) d p .
$$

In analogy to (12), we propose a cumulative past Rényi quantile entropy (CPRQE) that computes the uncertainty related to past. It is defined as

$$
\breve{\bar{\xi}}^{\alpha}(u)=\breve{\xi}^{\alpha}(X ; Q(u))=\frac{1}{(1-\alpha)} \log \left(\frac{1}{u^{\alpha}} \int_{0}^{u} p^{\alpha} q(p) d p\right) .
$$

When $u \longrightarrow 1$, (27) reduces to $\bar{\xi}^{\alpha}=\frac{1}{(1-\alpha)} \log \left(\int_{0}^{1} p^{\alpha} q(p) d p\right)$, a quantile version of cumulative Rényi entropy. Equation (27) can be written as, using (23)

$$
\breve{\breve{\xi}}^{\alpha}(u)=\frac{1}{(1-\alpha)} \log \left(\frac{1}{u^{\alpha}} \int_{0}^{u} p^{\alpha-1} \bar{M}(p) d p+\frac{1}{u^{\alpha}} \int_{0}^{u} p^{\alpha} \bar{M}^{\prime}(p) d p\right) .
$$

Applying integration by parts on the last term and simplify, we obtain

$$
u^{\alpha} e^{(1-\alpha) \breve{\xi}^{\alpha}(u)}-\bar{M}(u) u^{\alpha}=(1-\alpha) \int_{0}^{u} p^{\alpha-1} \bar{M}(p) d p .
$$

Differentiating (28) with respect to $u$ both sides, and using (21), (28) reduces to

$$
q(u)=\left\{\frac{\alpha}{u}+(1-\alpha) \breve{\bar{\xi}}^{\prime \alpha}(u)\right\} e^{(1-\alpha) \breve{\xi}^{\alpha}(u)},
$$

where prime denote the derivative with respect to $u$. Equation (29) provides a direct relationship between quantile density function $q(u)$ and $\breve{\xi}^{\alpha}(u)$. Therefore $\breve{\xi}^{\alpha}(u)$ uniquely determines the underlying distribution.

\section{Example 5}

If $X$ be a random variable having the Tukey lambda distribution with the quantile function $Q(u)=\frac{u^{\lambda}-(1-u)^{\lambda}}{\lambda}, 0 \leq$ $u \leq 1$; define for all nonzero lambda values. Then cumulative past Rényi quantile entropy, for Tukey lambda distribution is given as

$$
\breve{\bar{\xi}}^{\alpha}(u)=\frac{1}{(1-\alpha)} \log \left[\frac{1}{u^{\alpha}} \int_{0}^{u} p^{\alpha}\left\{p^{\lambda-1}+(1-p)^{\lambda-1}\right\} d p\right]
$$

which gives

$$
\breve{\breve{\xi}}^{\alpha}(u)=\frac{1}{(1-\alpha)} \log \left(\frac{u^{\lambda}}{\alpha+\lambda}+\frac{\beta_{u}(\alpha+1, \lambda)}{u^{\alpha}}\right),
$$

where $\beta_{u}(a, b)$ are known as the incomplete beta function defined as $\beta_{u}(a, b)=\int_{0}^{u} x^{a-1}(1-x)^{b-1} d x, a, b>$ $0, u>0$ respectively.

\section{Example 6}

When $X$ is distributed with quantile density function given by; $q(u)=K u^{\delta}(1-u)^{-(A+\delta)}$, where $\mathrm{K}, \delta$, and $\mathrm{A}$ are real constants. It contains several distribution which include the exponential when $\delta=0, A=1$, Pareto when $\delta=0, A<1$, rescaled beta when $\delta=0, A>1$, $\log \operatorname{logestic}$ distribution when $\delta=b-1, A=2$ and the life distribution proposed by Govindarajulu (1977) when $\delta=b-1, A=-b$, then

$$
\breve{\bar{\xi}}^{\alpha}(u)=\frac{1}{1-\alpha} \log \left(\frac{K \beta_{u}(1+\alpha+\delta, 1-A-\delta)}{u^{\alpha}}\right),
$$

where $\beta_{u}(a, b)$ are known as the incomplete beta function. 


\section{Characterization Results}

By considering a relationship between the dynamic cumulative residual Rényi quantile entropy $\breve{\xi}^{\alpha}(u)$ and the hazard quantile function $K(u)$. We characterize some lifetime distributions based on the quanlile entropy measure (17). We give the following theorem.

\section{Theorem 2}

Let $X$ be a random varible with hazard quantile function $K(u)$ for all $u \in(0,1)$. The relationship

$$
\breve{\xi}^{\alpha}(u)=\frac{1}{(1-\alpha)} \log c-\frac{1}{(1-\alpha)} \log K(u),
$$

where $c$ is constant, holds if and only if $X$ follow generalized Pareto distribution with quantile function $Q(u)=$ $\frac{b}{a}\left[(1-u)^{-\frac{a}{a+1}}-1\right] ; b>0, a>-1$.

\section{Proof}

The hazard quantile function of generalized Pareto distribution is $K(u)=\frac{(a+1)(1-u)^{\frac{a}{a+1}}}{b}$. Taking $c=\left(\frac{a+1}{\alpha(a+1)-a}\right)$ gives the if part of the theorem. To prove the only if part, consider (31) to be valid. Then

$$
\int_{u}^{1}(1-p)^{\alpha} q(p) d p=\frac{c(1-u)^{\alpha}}{K(u)}
$$

Using (4), we have

$$
\int_{u}^{1}(1-p)^{\alpha} q(p) d p=c(1-u)^{1+\alpha} q(u)
$$

Differentiating both side with respect to $u$ and after some algebraic simplification, we have

$$
\frac{q^{\prime}(u)}{q(u)}=\left(\frac{c(\alpha+1)-1}{c}\right) \frac{1}{(1-u)} .
$$

This gives

$$
q(u)=A(1-u)^{\frac{1}{c}-(\alpha+1)},
$$

where $A$ is the constant. Substituting the value of $c$, this gives

$$
q(u)=A(1-u)^{-\frac{2 a}{a+1}},
$$

which characterizes the generalized Pareto distribution. Hence proved.

Next we extend the result to more general case where $\mathrm{c}$ is a function of $u$.

Theorem 3

Let $X$ be a nonnegative absolutely continuous random variable with hazard quantile function $K(u)$ and the $D C R R Q E \breve{\xi}^{\alpha}(u)$ is

$$
\breve{\xi}^{\alpha}(u)=\frac{1}{(1-\alpha)} \log c(u)-\frac{1}{(1-\alpha)} \log K(u), u>0,
$$

then

$$
q(u)=\exp \left(\frac{\int_{0}^{u} \frac{-d u}{(1-u) c(u)}}{(1-u)^{\alpha+1} c(u)}\right) .
$$

Proof

Let (32) be valid. Then

$$
\frac{1}{1-\alpha} \log \left\{\frac{1}{(1-u)^{\alpha}} \int_{u}^{1}(1-p)^{\alpha} q(p) d p\right\}=\frac{1}{1-\alpha} \log \left(\frac{c(u)}{K(u)}\right) .
$$


Substituting the value of K(u) from (4), we have

$$
\int_{u}^{1}(1-p)^{\alpha} q(p) d p=c(u) q(u)(1-u)^{\alpha+1} .
$$

Differentiating both side with respect to $u$ and after some algebraic simplifying, we have

$$
\frac{q^{\prime}(u)}{q(u)}=\frac{(\alpha+1)}{(1-u)}-\frac{1}{(1-u) c(u)}-\frac{c^{\prime}(u)}{c(u)},
$$

where prime denotes derivative with respect to $u$. Integrating with respect to $u$ both side between 0 to $u$ in the above expression and simplifying, we obtain

$$
\log \left(c(u)(1-u)^{\alpha+1} q(u)\right)=-\int_{0}^{u} \frac{1}{(1-u) c(u)} .
$$

In particular, if $c(u)=a u+b$ and $\mathrm{a}, \mathrm{b} \geq 0$ then above gives

$$
q(u)=\frac{1}{(1-u)^{\alpha+1}(a u+b)}\left(\frac{a u+b}{b(1-u)}\right)^{\frac{-1}{a+b}} .
$$

Further we note that expression (34) for a $=0$, gives the characterization result given by Theorem (31).

The following theorem gives another characterization of generalized Pareto distribution using the relationship between $\breve{\xi}^{\alpha}(u)$ and mean residual quantile function $M(u)$, the proof of which follow on the same line as given by Theorem (3), hence omitted.

Theorem 4

Let $X$ be a random varible with mean residual quantile function $M(u)$ for all $u \in(0,1)$. The relationship

$$
\breve{\xi}^{\alpha}(u)=\frac{1}{(1-\alpha)} \log c+\frac{1}{(1-\alpha)} \log M(u),
$$

where $c$ is constant, holds if and only if $X$ follow generalized Pareto distribution with quantile function $Q(u)=$ $\frac{b}{a}\left[(1-u)^{-\frac{a}{a+1}}-1\right]$.

Theorem 5

For a nonnegative random variable $X$, the relationship

$$
(1-\alpha) \breve{\xi}^{\alpha}(u)=\frac{-C}{1-u},
$$

where $\mathrm{C}$ is constant, holds for all $u \in(0,1)$ if and only if $X$ follows

(i) uniform distribution for $C=1$

(ii) exponential distribution for $C=0$

(iii) Pareto I distribution for $C=\frac{-1}{a}$

Proof

The necessity part follows from Table (14). For sufficiency part, let us assume that the relationship (36) holds. From equation (17) and (20), we have

$$
-\frac{(1-u)^{\alpha} q(u)}{\int_{u}^{1}(1-p)^{\alpha} q(p) d p}+\frac{\alpha}{1-u}=-\frac{C}{1-u} .
$$


After some algebraic simplification, we obtain

$$
q(u)(1-u)^{\alpha+1}=(\alpha+C) \int_{u}^{1}(1-p)^{\alpha} q(p) d p .
$$

Differentiating both side with respect to $u$ and after simplification, we get

$$
-q(u)(1-u)^{\alpha}(\alpha+1)+(1-u)^{\alpha+1} q^{\prime}(u)=-(\alpha+C)(1-u)^{\alpha} q(u),
$$

or equivalently

$$
\frac{q^{\prime}(u)}{q(u)}=\frac{1-C}{1-u}
$$

This gives

$$
q(u)=(1-u)^{C-1} e^{K},
$$

where $\mathrm{K}$ is the constant of integration. Now, if $\mathrm{C}=1$ and $K=\log (b-a) ; b>a$, which implies that $Q(u)=$ $a+(b-a) u$. Thus, we have the uniform distribution. If $C=0$ and $K=-\log \lambda ; \lambda \geq 0$, which implies that $Q(u)=$ $-\lambda^{-1} \log (1-u)$. Thus, we have the exponential distribution with parameter $\lambda$. If, $C=\frac{-1}{a}$ and $K=\log \left(\frac{b}{a}\right)$, that $a$ and $b$ are positive constants, we have $Q(u)=b(1-u)^{-\frac{1}{a}}$. This means, we have the Pareto I distribution.

In the following theorem we characterize the power distribution, when CPRQE $\breve{\xi}^{\alpha}(u)$ is expressed in terms of reverse hazard quantile function $\bar{K}(u)(21)$.

\section{Theorem 6}

Let $X$ be a nonnegative continuous random variable with reverse hazard quantile function $\bar{K}(u)$ for all $u \in(0,1)$ and cumulative past Rényi quantile entropy $\breve{\bar{\xi}}^{\alpha}(u)$ given by

$$
\breve{\breve{\xi}}^{\alpha}(u)=\frac{1}{(1-\alpha)} \log c-\frac{1}{(1-\alpha)} \log \bar{K}(u) .
$$

If and only if $X$ has power distribution function.

Proof

The reverse hazard quantile function of power distribution is $\bar{K}(u)=\frac{b u \frac{-1}{b}}{a}$. Taking $c=\frac{b}{b \alpha+1}$ gives the if part of the theorem. To prove the only if part, consider (37) to be valid. Using (27), it gives

$$
\frac{\int_{0}^{u} p^{\alpha} q(p) d p}{u^{\alpha}}=\frac{c}{\bar{K}(u)} .
$$

Substituting $\bar{K}(u)=\frac{1}{u q(u)}$, gives

$$
\int_{0}^{u} p^{\alpha} q(p) d p=c u^{\alpha+1} q(u)
$$

Differentiating both side with respect to $u$ and simplifying, this reduces to

$$
\frac{q^{\prime}(u)}{q(u)}=\left(\frac{1-c(\alpha+1)}{c}\right) \frac{1}{u} .
$$

Which lead to

$$
q(u)=A u^{\frac{1}{c}-(\alpha+1)},
$$

where A is a constant. Which characterizes the power distribution for $c=\frac{b}{b \alpha+1}$.

Next we characterize the lifetime models when CPRQE (27) is expressed in terms of quantile version of mean inactivity time $\bar{M}(u)$. The proof follows on the same line as Theorem (3.5), hence omitted. 
Theorem 7

Let $X$ be a nonnegative continuous random variable with mean residual quantile function $\bar{M}(u)$ for all $u \in(0,1)$ and cumulative past Rényi quantile entropy $\breve{\xi}^{\alpha}(u)$ given by

$$
\breve{\breve{\xi}}^{\alpha}(u)=\frac{1}{(1-\alpha)} \log c+\frac{1}{(1-\alpha)} \log \bar{M}(u),
$$

where $\mathrm{c}$ is constant. If and only if $X$ has power distribution function.

\section{DCRRQE of Order Statistics $X_{i: n}$}

Suppose $X_{1}, X_{2}, \ldots, X_{n}$ be a random sample from a population with probability density function $f$ and cumulative distribution function $F($.$) and let as X_{1: n} \leq X_{2: n} \leq \ldots X_{n: n}$ be the order statistics obtained by arranging the preceding random sample in increasing order of magnitude. Then the pdf of $i^{\text {th }}$ order statistics $X_{i: n}$ is given by

$$
f_{i: n}(x)=\frac{1}{B(i, n-i+1)}(F(x))^{i-1}(\bar{F}(x))^{n-i} f(x),
$$

where $B(a, b)=\int_{0}^{1} x^{a-1}(1-x)^{b-1} d x ; a, b>0$, be the beta function. The corresponding quantile-based density function of is $f_{i: n}(x)$ becomes

$$
f_{i: n}(u)=f_{i: n}(Q(u))=\frac{u^{i-1}(1-u)^{n-i}}{B(i, n-i+1) q(u)}
$$

Sunoj et al. (2017) introduced a quantile-based entropy of order statistics and studied its properties. Order statistics play an important role in system reliability. These statistics have been used in a wide range of problems, including robust statistical estimation, detection of outliers, characterization of the probability distribution and goodness-offit tests, analysis of censored samples, reliability analysis, quality control and strength of materials, see, for details, Arnold et al. (1992), David and Nagaraja (2003), and references therein. Fashandi and Ahmadi (2012) have derived certain characterizations for symmetric distributions based on Rényi entropy of order statistics, $k$ record statistics and the FGM family of bivariate distributions. Gupta et al. (2014) proved some characterization result based on dynamic entropy of order statistics.

Abbasnejad and Arghami (2010) defined Rényi entropy of order $\alpha$ of $i^{\text {th }}$ order statistics $X_{i: n}$ as

$$
H^{\alpha}\left(X_{i: n}\right)=\frac{1}{(1-\alpha)} \log \left\{\int_{0}^{\infty}\left(f_{i: n}(x)^{\alpha} d x\right\}, \alpha>0, \alpha \neq 1 .\right.
$$

Kumar and Nirdesh (2019) proposed quantile-based Rényi entropy of $X_{i: n}$, which is given as

$$
S_{X_{i: n}}^{\alpha}=\frac{1}{(1-\alpha)} \log \int_{0}^{1}\left(\frac{1}{B(i, n-i+1)}\right)^{\alpha} p^{\alpha(i-1)}(1-p)^{\alpha(n-i)}(q(u))^{1-\alpha} d p .
$$

and studied some properties of it. Unlike (38), $S_{X_{i: n}}^{\alpha}$ will be more useful in cases we do not have a tractable distribution function but have a closed quantile function. In analogy with (12), the cumulative residual Rényi entropy of $i^{t h}$ order statistic $X_{i: n}$ is defined as

$$
\begin{aligned}
\xi_{X_{i: n}}^{\alpha} & =\frac{1}{(1-\alpha)} \log \int_{0}^{\infty} \bar{F}_{i: n}^{\alpha}(x) d x \\
& =\frac{1}{(1-\alpha)} \log \int_{0}^{\infty}\left(\frac{\bar{\beta}_{F(x)}(i, n-i+1)}{\beta(i, n-i+1)}\right)^{\alpha} d x
\end{aligned}
$$


where $\bar{F}_{i: n}(x)=\frac{\bar{\beta}_{F(x)}(i, n-i+1)}{\beta(i, n-i+1)}$ is the survival function of the $i^{t h}$ order statistics. The cumulative residual Rényi quantile entropy of order statistics (39) becomes

$$
\breve{\xi}_{X_{i: n}}^{\alpha}=\frac{1}{(1-\alpha)} \log \int_{0}^{1}\left(\frac{\bar{\beta}_{p}(i, n-i+1)}{\beta(i, n-i+1)}\right)^{\alpha} q(p) d p,
$$

where $\frac{\beta_{u}(i, n-i+1)}{\beta(i, n-i+1)}$ is the quantile form of survival function $\bar{F}_{i: n}(x)$. In system reliability, first order statistic represents the lifetime of a series system while the $n^{\text {th }}$ order statistic measure the lifetime of a parallel system. For a series system $(i=1)$, we have

$$
\breve{\xi}_{X_{1: n}}^{\alpha}=\frac{1}{(1-\alpha)} \log \left(\int_{0}^{1}(1-p)^{n \alpha} q(p) d p\right) .
$$

For the parallel system $(i=n)$, we have

$$
\breve{\xi}_{X_{n: n}}^{\alpha}=\frac{1}{(1-\alpha)} \log \left(\int_{0}^{1}\left(1-p^{n}\right)^{\alpha} q(p) d p\right) .
$$

The residual lifetime of a system when it is still operating at time $\mathrm{t}$, is $\left(X_{t}=X-t \mid X>t\right)$ which has the probability density function $f(x, t)=\frac{f(x)}{\bar{F}(t)}, x \geq t>0$. Thapliyal and Taneja (2015) studied dynamic cumulative residual Rényi entropy (DCRRE) measure for the $X_{i: n}$, which is given by

$$
H^{\alpha}\left(X_{i: n} ; t\right)=\frac{1}{(1-\alpha)} \log \left(\int_{t}^{\infty} \frac{\bar{F}_{i: n}^{\alpha}(x)}{\bar{F}_{i: n}^{\alpha}(t)} d x\right) .
$$

For $i^{t h}$ order statistics $X_{i: n}$, the quantile version of DCRRE is

$$
\begin{aligned}
\breve{\xi}_{X_{i: n}}^{\alpha}(u)=\breve{\xi}_{X_{i: n}}^{\alpha}(Q(u)) & =\frac{1}{(1-\alpha)} \log \left\{\int_{u}^{1}\left(\frac{\bar{\beta}_{p}(i, n-i+1)}{\beta(i, n-i+1)}\right)^{\alpha}\left(\frac{\beta(i, n-i+1)}{\bar{\beta}_{u}(i, n-i+1)}\right)^{\alpha} q(p) d p\right) \\
= & \frac{1}{(1-\alpha)} \log \left\{\frac{1}{\left(\bar{\beta}_{u}(i, n-i+1)\right)^{\alpha}} \int_{u}^{1}\left(\left(\bar{\beta}_{p}(i, n-i+1)\right)^{\alpha}\right) q(p) d p\right\}
\end{aligned}
$$

where $\frac{\beta_{u}(i, n-i+1)}{\beta(i, n-i+1)}$ is the quantile form of survival function $\bar{F}_{i: n}(x)$ and $\bar{\beta}_{x}(a, b)=\int_{x}^{1} u^{a-1}(1-u)^{b-1} d u, 0<x<$ 1 , is the incomplete beta function, see David and Nagaraja (2003). An equivalent representation of (43) is of the form

$$
\exp ^{(1-\alpha) \breve{\xi}_{X_{i: n}}^{\alpha}(u)}\left(\bar{\beta}_{u}(i, n-i+1)\right)^{\alpha}=\int_{u}^{1}\left(\bar{\beta}_{p}(i, n-i+1)\right)^{\alpha} q(p) d p .
$$

Differentiating (43) with respect to $u$ both sides and after some algebraic simplification,we obtain

$$
q(u)=\left\{\frac{\alpha u^{i-1}(1-u)^{n-i}}{\bar{\beta}_{u}(i, n-i+1)}-(1-\alpha) \breve{\xi}_{X_{i: n}^{\alpha}}^{\alpha}(u)\right\} e^{(1-\alpha) \breve{\xi}_{X_{i: n}}^{\alpha}(u)} .
$$

Equation (44) provide a direct relationship between quantile density function $q(u)$ and $\breve{\xi}_{X_{i: n}}^{\alpha}(u)$ which show that $\breve{\xi}_{X_{i: n}}^{\alpha}(u)$ uniquely determines the underlying distribution.

In system reliability, the minimum and maximum are examples of extreme order statistics and are defined by $X_{1: n}=\min \left\{X_{1}, X_{2}, \ldots, X_{n}\right\}$ and $X_{n: n}=\max \left\{X_{1}, X_{2}, \ldots, X_{n}\right\}$. The extreme $X_{1: n}$ and $X_{n: n}$ are of special interest in many practical problems of distribution analysis. The extremes aries in the statistical study of floods and droughts, as well as in problems of breaking strength and fatigue failure. Substituting $(i=1)$ in (43) then the 
$D C R R Q E$ of first order statistic $X_{1: n}$, given as

$$
\begin{gathered}
\breve{\xi}_{X_{i: n}}^{\alpha}(u)=\frac{1}{(1-\alpha)} \log \left\{\frac{1}{\left(\bar{\beta}_{u}(1, n)\right)^{\alpha}} \int_{u}^{1}\left(\bar{\beta}_{p}(1, n)\right)^{\alpha} q(p) d p\right\} . \\
=\frac{1}{(1-\alpha)} \log \left\{\frac{1}{(1-u)^{n \alpha}} \int_{u}^{1}(1-p)^{n \alpha} q(p) d p\right\} .
\end{gathered}
$$

The DCRRQE for sample maxima of order statistic $X_{n: n}$ can be obtained from (43) by taking $(i=n)$, as

$$
\begin{aligned}
\breve{\xi}_{X_{n: n}}^{\alpha}(u) & =\frac{1}{(1-\alpha)} \log \left\{\frac{1}{\left(\bar{\beta}_{u}(n, 1)\right)^{\alpha}} \int_{u}^{1}\left(\bar{\beta}_{p}(n, 1)\right)^{\alpha} q(p) d p\right\} \\
= & \frac{1}{(1-\alpha)} \log \left\{\frac{1}{\left(1-u^{n}\right)^{\alpha}} \int_{u}^{1}\left(1-p^{n}\right)^{\alpha} q(p) d p\right\} .
\end{aligned}
$$

For various specific univariate continuous distributions, the expression (45) is evaluated as given below in Table 38.

Table 4.1 Quantile function and $\breve{\xi}_{X_{1: n}}^{\alpha}(u)$ for various lifetime distributions

\begin{tabular}{|c|c|c|}
\hline Distribution & Quantile function $Q(u)$ & $\breve{\xi}_{X_{1: n}}^{\alpha}(u)$ \\
\hline Uniform & $a+(b-a) u$ & $\frac{1}{1-\alpha} \log \left\{\frac{(b-a)(1-u)}{n \alpha+1}\right\}$ \\
Exponential & $-\lambda^{-1} \log (1-u)$ & $\frac{1}{1-\alpha} \log \left\{\frac{1}{n \alpha \lambda}\right\}$ \\
Pareto I & $b(1-u)^{-\frac{1}{a}}$ & $\frac{1}{1-\alpha} \log \left\{\frac{b(1-u)^{\frac{-1}{a}}}{n a \alpha-1}\right\}$ \\
Folded Cramer & $\frac{u}{\theta(1-u)}$ & $\frac{1}{1-\alpha} \log \left\{\frac{1}{\theta(n \alpha-1)(1-u)}\right\}$ \\
Generalized Pareto & $\frac{b}{a}\left[(1-u)^{-\frac{a}{a+1}}-1\right]$ & $\frac{1}{1-\alpha} \log \left\{\frac{b(1-u)^{\frac{-a}{a+1}}}{(n \alpha a+n \alpha-a)}\right\}$ \\
Finite Range & $b\left(1-(1-u)^{\frac{1}{a}}\right)$ & $\frac{1}{1-\alpha} \log \left\{\frac{b(1-u)^{\frac{1}{a}}}{(n \alpha a+1)}\right\}$ \\
Log logestic & $\frac{1}{a}\left(\frac{u}{1-u}\right)^{\frac{1}{b}}$ & $\frac{1}{1-\alpha} \log \left\{\frac{\bar{\beta}_{u}\left[b, n \alpha-\frac{1}{b}\right]}{a b(1-u)^{n \alpha}}\right\}$ \\
Generalized lambda & $\lambda_{1}+\frac{1}{\lambda_{2}}\left(\frac{u^{\lambda_{3}-1}}{\lambda_{3}}-\frac{(1-u)^{\lambda_{4}}-1}{\lambda_{4}}\right)$ & $\frac{1}{1-\alpha} \log \left\{\frac{\left(\lambda_{3}-1\right)}{\lambda_{2} \lambda_{3}(1-u)^{n \alpha}} \bar{\beta}_{u}\left(\lambda_{3}-1, n \alpha+1\right)+\frac{(1-u)^{\lambda_{4}}}{\lambda_{2}\left(n \alpha+\lambda_{4}\right)}\right\}$ \\
Skew lambda & $\alpha u^{\lambda}-(1-u)^{\lambda}$ & $\frac{1}{1-\alpha} \log \left\{\frac{\alpha \lambda \bar{\beta}_{u}(\lambda, n \alpha+1)}{(1-u)^{n \alpha}}+\frac{\lambda(1-u)}{n \alpha+\lambda}\right\}$ \\
Govindarajulu's & $a\left((b+1) u^{b}-b u^{(b+1)}\right)$ & $\frac{1}{1-\alpha} \log \left(\frac{a b(b+1) \bar{\beta}_{u}(b, n \alpha+2)}{(1-u)^{n \alpha}}\right)$ \\
Tukey lambda & $\frac{u^{\lambda}-(1-u)^{\lambda}}{\lambda}$ & $\frac{1}{1-\alpha} \log \left(\frac{\bar{\beta}_{u}(\lambda, n \alpha+1)}{(1-u)^{n \alpha}}+\frac{(1-u)^{\lambda}}{n \alpha+\lambda}\right)$ \\
\hline
\end{tabular}

Below we see how the monotonicity of $\breve{\xi}_{X_{1: n}}^{\alpha}(u)$ is affected by increasing transformation. The following lemma help us to prove the results on monotonicity of $\breve{\xi}_{X_{1: n}}^{\alpha}(u)$.

\section{Lemma 1}

Let $f(u, x): \Re_{+}^{2} \longrightarrow \Re_{+}$and $g: \Re_{+} \longrightarrow \Re_{+}$be any two functions. If $\int_{u}^{\infty} f(u, x) d x$ is increasing and $g(u)$ is increasing (decreasing) in $u$, then $\int_{u}^{\infty} f(u, x) g(x) d x$ is increasing (decreasing) in $u$, provided the integrals exist.

\section{Theorem 8}

Let $X$ be a nonnegative and continuous random variable with quantile function $Q_{X}($.$) and quantile density function$ $q_{X}($.$) . Define Y=\phi(X)$, where $\phi($.$) is a nonnegative, increasing and convex (concave) function.$

(i) For $0<\alpha<1, \breve{\xi}_{Y_{1: n}^{\alpha}}^{\alpha}(u)$ is increasing (decreasing) in $u$ whenever $\breve{\xi}_{X_{1: n}}^{\alpha}(u)$ is increasing (decreasing) in $u$.

(ii)For $\alpha>1, \breve{\xi}_{Y_{1: n}}^{\alpha}(u)$ is decreasing (increasing) in $u$ whenever $\breve{\xi}_{X_{1: n}}^{\alpha}(u)$ is increasing (decreasing) in $u$. 
Proof

(i) The probability density function of $Y=\phi(X)$ is $g(y)=\frac{f\left(\phi^{-1}(y)\right)}{\phi^{\prime}\left(\phi^{-1}(y)\right)}$; hence density quantile function is $g\left(Q_{Y}(u)\right)=\frac{1}{q_{Y}(u)}=\frac{f(Q(u))}{\phi^{\prime} Q(u)}=\frac{1}{q_{X}(u) \phi^{\prime}\left(Q_{X}(u)\right)}$. Thus we have

$$
\begin{aligned}
\breve{\xi}_{Y_{1: n}}^{\alpha}(u) & =\frac{1}{(1-\alpha)} \log \left(\frac{1}{(1-u)^{n \alpha}} \int_{u}^{1}(1-p)^{n \alpha} q_{Y}(p) d p\right) \\
= & \left.\frac{1}{(1-\alpha)} \log \left(\frac{1}{(1-u)^{n \alpha}} \int_{u}^{1}(1-p)^{n \alpha} q_{X}(p) \phi^{\prime}\left(Q_{X}(p)\right)\right) d p\right)
\end{aligned}
$$

From the given condition we have

$$
\frac{1}{(1-\alpha)} \log \left(\frac{1}{(1-u)^{n \alpha}} \int_{u}^{1}(1-p)^{n \alpha} q_{X}(p) d p\right) \text { is increasing in } u,
$$

which gives that

$$
\log \left(\frac{1}{(1-u)^{n \alpha}} \int_{u}^{1}(1-p)^{n \alpha} q_{Y}(p) d p\right) \text { is increasing in } u
$$

We can rewritten as

$$
(1-\alpha) \breve{\xi}_{Y_{1: n}}^{\alpha}(u)=\log \left(\frac{1}{(1-u)^{n \alpha}} \int_{u}^{1}(1-p)^{n \alpha} q_{X}(p) \phi^{\prime}\left(Q_{X}(p)\right) d p\right)
$$

Since $0<\alpha<1$ and $\phi$ is nonnegative, increasing convex (concave), we have $\left[\phi^{\prime}(Q(p))\right]^{1-\alpha}$ is increasing (decreasing) and is nonnegative. Hence by Lemma 4.1, (48) is increasing (decreasing). This prove (i). When $\alpha>1$, $\left[\phi^{\prime}(Q(p))\right]^{1-\alpha}=\frac{1}{\left[\phi^{\prime}(Q(p))\right]^{\alpha-1}}$ is decreasing in $p$, since $\phi$ is increasing and convex. Hence we have

$$
\breve{\xi}_{Y_{1: n}}^{\alpha}(u)=\frac{1}{(1-\alpha)} \log \left(\frac{1}{(1-u)^{n \alpha}} \int_{u}^{1}(1-p)^{n \alpha} q_{Y}(p) d p\right),
$$

is decreasing (increasing) in $u$. Hence prove.

Remark 1

For any absolutely continuous random variable $X$, define $Y=a X+b, a \geq 0, b \geq 0$ then

$$
\breve{\xi}_{Y_{1: n}}^{\alpha}(u)=\frac{1}{(1-\alpha)} \log a+\breve{\xi}_{X_{1: n}}^{\alpha}(u)
$$

\section{Example 7}

A lambda family of distribution that is of interest in reliability is the Davis Distribution proposed by Hankin and Lee (2006) with quantile function $Q(u)=C u^{\lambda_{1}}(1-u)^{-\lambda_{2}}, 0<u<1, C, \lambda_{1}, \lambda_{2} \geq 0$. This is a flexible family for right skewed on non negative data that provide good approximation to the exponential, gamma, lognormal and weibull Distribution,. A special feature of these families is that they are expressed in terms of quantile functions for which distribution function are not available in closed form to facilitate the conventional analysis. The DCRRQE entropy of sample minima for Davis Distribution is given by

$$
\breve{\xi}_{X_{1: n}}^{\alpha}(u)=\frac{1}{(1-\alpha)} \log \left(C \lambda_{1} \frac{\bar{\beta}_{u}\left(\lambda_{1}, n \alpha-\lambda_{2}+1\right)}{(1-u)^{n \alpha}}+C \lambda_{2} \frac{\bar{\beta}_{u}\left(\lambda_{1}+1, n \alpha-\lambda_{2}\right)}{(1-u)^{n \alpha}}\right) .
$$

As $\lambda_{1} \longrightarrow 0$ (50) reduces to $\breve{\xi}_{X_{1: n}}^{\alpha}(u)=\frac{1}{(1-\alpha)} \log \left(\frac{C \lambda_{2}}{(1-u)^{\lambda}\left(n \alpha-\lambda_{2}\right)}\right)$, corresponding to Pareto I distribution. Also, as $\lambda_{2} \longrightarrow 0,(50)$ reduces to $\breve{\xi}_{X_{1: n}}^{\alpha}(u)=\frac{1}{(1-\alpha)} \log \left(\frac{C \lambda_{1} \bar{\beta}_{u}\left(\lambda_{1}, n \alpha+1\right)}{(1-u)^{n \alpha}}\right)$, corresponding to power distribution. 
Next, we obtain the characterization result based on first (minima) order statistic and last (maxima) order statistic in a random sample $X_{1}, X_{2}, \ldots, X_{n}$ of size $\mathrm{n}$ from positive and continuous random variable $X$.

\section{Theorem 9}

Let $X_{1: n}$ denote the first order statistic with survival function $\bar{F}_{1: n}(x)$ and hazard quantile function $K_{X_{1: n}}(u)$. Then the relationship

$$
\breve{\xi}_{X_{1: n}}^{\alpha}(u)=\frac{1}{(1-\alpha)} \log c-\frac{1}{(1-\alpha)} \log K_{X_{1: n}}(u)
$$

where $c$ is constant, holds for all $u \in(0,1)$ if and only if $X$ is distributed as generalized Pareto distribution with quantile function $Q(u)=\frac{b}{a}\left[(1-u)^{-\frac{a}{a+1}}-1\right] ; b>0, a>-1$.

Proof

Consider (51) to be valid. Then

$$
\int_{u}^{1}(1-p)^{n \alpha} q(p) d p=\frac{c(1-u)^{n \alpha}}{K_{X_{1: n}}(u)}
$$

Substituting $K_{X_{1: n}}(u)=\frac{f_{1: n}(Q(u))}{1-F(Q(u))^{n}}=\frac{n}{(1-u) q(u)}$ and simplifying, it gives

$$
n \int_{u}^{1}(1-p)^{n \alpha} q(p) d p=c q(u)(1-u)^{n \alpha+1} .
$$

Differentiating with respect to $u$ both side and after some algebraic simplification, we have

$$
\frac{q^{\prime}(u)}{q(u)}=\left(\frac{n-c(n \alpha+1)}{c}\right) \frac{1}{(1-u)} .
$$

This gives

$$
q(u)=A(1-u)^{\frac{n}{c}-(n \alpha+1)},
$$

where $A$ is constant, which characterizes the generalized Pareto distribution. The only if part of the theorem is easy to proved.

\section{Corollary 1}

Let $X_{1: n}$ denote the first order statistic with survival function $\bar{F}_{1: n}(x)$ and hazard quantile function $K_{X_{1: n}}(u)$ for all $u \in(0,1)$. Then the relationship

$$
\breve{\xi}_{X_{1: n}}^{\alpha}(u)=\frac{1}{(1-\alpha)} \log c-\frac{1}{(1-\alpha)} \log K_{X_{1: n}}(u) .
$$

holds, if and only if for (i) $c=\frac{1}{\alpha}, X$ follows exponential distribution (ii) $c<\frac{1}{\alpha}, X$ follows Pareto I distribution (iii) $c>\frac{1}{\alpha}, X$ follows finite range distribution.

Theorem 10

Let $X_{1: n}$ denote the first order statistic with survival function $\bar{F}_{1: n}(x)$. Then the relationship

$$
(1-\alpha) \breve{\xi}_{X_{1: n}}^{\alpha}(u)=\frac{-C}{1-u}
$$

where $\mathrm{C}$ is constant, holds for all $u \in(0,1)$ if and only if $X$ is distributed as

(i) uniform distribution for $C=1$

(ii) exponential distribution for $C=0$

(iii) Pareto I distribution for $C=\frac{-1}{a}$ 
Proof

The necessity part follows from Table (38). For sufficient part, let us assume that the relationship (52) holds. From (45) and (47), we have

$$
-\frac{(1-u)^{n \alpha} q(u)}{\int_{u}^{1}(1-p)^{n \alpha} q(u) d p}+\frac{n \alpha}{1-u}=-\frac{C}{1-u} .
$$

After simplifying, we have

$$
q(u)(1-u)^{n \alpha+1}=(n \alpha+C) \int_{u}^{1}(1-p)^{n \alpha} q(p) d p .
$$

Differentiating both side with respect to $u$ and after some algebraic simplification, we get

$$
-q(u)(1-u)^{n \alpha}(n \alpha+1)+(1-u)^{n \alpha+1} q^{\prime}(u)=-(n \alpha+C)(1-u)^{n \alpha} q(u),
$$

or equivalently

$$
\frac{q^{\prime}(u)}{q(u)}=\frac{1-C}{1-u}
$$

Which lead to

$$
q(u)=(1-u)^{C-1} e^{K},
$$

where $\mathrm{K}$ is the constant of integration. Now, if $\mathrm{C}=1$ and $K=\log (b-a) ; b>a$, which implies that $Q(u)=$ $a+(b-a) u$. Thus, we have the uniform distribution. If $C=0$ and $K=-\log \lambda ; \lambda \geq 0$, which implies that $Q(u)=$ $-\lambda^{-1} \log (1-u)$. Thus, we have the exponential distribution with parameter $\lambda$. If, $C=\frac{-1}{a}$ and $K=\log \left(\frac{b}{a}\right)$, that $a$ and $b$ are positive constants, we have $Q(u)=b(1-u)^{-\frac{1}{a}}$. This means, we have the Pareto I distribution.

Theorem 11

Let $X_{1: n}$ denote the first order statistic with survival function $\bar{F}_{1: n}(x)$ and hazard quantile function $K_{X_{1: n}}(u)$. Then the relationship given by

$$
(n \alpha+1-C) e^{(1-\alpha) \breve{\xi}_{X_{1: n}}^{\alpha}(u)}=\frac{n}{K_{X_{1: n}}(u)},
$$

where $\mathrm{C}$ is constant, holds for all $u \in(0,1)$ if and only if $X$ is distributed

(i) uniform distribution for $C=0$

(ii) exponential distribution for $C=1$

(iii) Pareto I distribution for $C=1+\frac{1}{a}$

Proof

The necessity part follows from Table (38). For sufficiency part, let us assume that the relationship (53) holds. Substituting $K_{X_{1: n}}(u)=\frac{n}{(1-u) q(u)}$ and equation(45), we have

$$
(n \alpha+1-C) \frac{\int_{u}^{1}(1-p)^{n \alpha} q(u) d p}{(1-u)^{n \alpha}}=(1-u) q(u) .
$$

Differentiating both side with respect to $u$ and after some algebraic simplification, we get

$$
(n \alpha+1-C)(1-u)^{n \alpha} q(u)=(n \alpha+1)(1-u)^{n \alpha} q(u)-(1-u)^{n \alpha+1} q^{\prime}(u) .
$$

By the above equation, we have

$$
\frac{q^{\prime}(u)}{q(u)}=\frac{C}{1-u}
$$

This gives

$$
q(u)=(1-u)^{-C} e^{A},
$$

where $A$ is the constant of integration. Now, for $C=0, C=1$ and $C=1+\frac{1}{a}$ and with appropriate values of $A$, we obtain the desired result. 
For the sample minima $X_{1: n}$, the relationship (6) becomes $(1-u) q(u)=n M_{X_{1: n}}(u)-(1-u) M_{X_{1: n}}^{\prime}(u)$. We state a characterization result using the relationship between $\breve{\xi}_{X_{1: n}}^{\alpha}(u)$ and $M_{X_{1: n}}(u)$, the proof of which follow on the same line as given by Theorem (3.1), hence omitted.

Theorem 12

Let $X_{1: n}$ denote the first order statistic with survival function $\bar{F}_{1: n}(x)$ and mean residual quantile function $M_{X_{1: n}}(u)$ for all $u \in(0,1)$. Then the relationship given by

$$
\breve{\xi}_{X_{1: n}}^{\alpha}(u)=\frac{1}{(1-\alpha)} \log c+\frac{1}{(1-\alpha)} \log M_{X_{1: n}}(u) .
$$

where $c$ is constant, holds if and only if $X$ has generalized Pareto distribution with quantile function $Q(u)=$ $\frac{b}{a}\left[(1-u)^{-\frac{a}{a+1}}-1\right] ; b>0, a>-1$.

Corollary 2

Let $X_{1: n}$ denote the first order statistic with survival function $\bar{F}_{1: n}(x)$ and mean residual quantile function $M_{X_{1: n}}(u)$. Then the relationship

$$
\breve{\xi}_{X_{1: n}}^{\alpha}(u)=\frac{1}{(1-\alpha)} \log c+\frac{1}{(1-\alpha)} \log M_{X_{1: n}}(u) .
$$

where $c$ is constant, holds for all $u \in(0,1)$ if and only if for (i) $c=\frac{1}{\alpha}, X$ follows exponential distribution (ii) $c<\frac{1}{\alpha}, X$ follows Pareto I distribution (iii) $c>\frac{1}{\alpha}, X$ follows finite range distribution.

Let $X_{n: n}$ be the largest order statistic in a random sample of size $\mathrm{n}$ from an absolutely continuous nonnegative random variable $X$. Then the dynamic cumulative past Renyi entropy for sample maxima is as follow

$$
\bar{\xi}^{\alpha}\left(X_{n: n} ; t\right)=\frac{1}{(1-\alpha)} \log \left(\int_{0}^{t} \frac{F_{n: n}^{\alpha}(x)}{F_{n: n}^{\alpha}(t)} d x\right) .
$$

The quantile-based dynamic cumulative past Rényi entropy for $X_{n: n}$ can be expressed as

$$
\breve{\bar{\xi}}_{X_{n: n}}^{\alpha}(u)=\breve{\bar{\xi}}_{X_{n: n}}^{\alpha}(Q(u))=\frac{1}{(1-\alpha)} \log \left(\frac{1}{u^{n \alpha}} \int_{0}^{u} p^{n \alpha} q(p) d p\right) .
$$

For some specific univariate continuous distributions, the expression (56) is evaluated as given below in Table 4.2.

Table 4.2 Quantile function and $\breve{\xi}_{X_{n: n}}^{\alpha}(u)$ for some lifetime distributions

\begin{tabular}{|c|c|c|}
\hline Distribution & Quantile function $Q(u)$ & \multicolumn{1}{c|}{$\overline{\bar{\xi}}_{X_{n: n}}^{\alpha}(u)$} \\
\hline Uniform & $a+(b-a) u$ & $\frac{1}{1-\alpha} \log \left\{\frac{(b-a) u}{n \alpha+1}\right\}$ \\
Power & $a u^{\frac{1}{b}}$ & $\frac{1}{1-\alpha} \log \left\{\frac{a u^{\frac{1}{b}}}{n b \alpha+1}\right\}$ \\
Generalized lambda & $\lambda_{1}+\frac{1}{\lambda_{2}}\left(\frac{u^{\lambda_{3}-1}}{\lambda_{3}}-\frac{(1-u)^{\lambda_{4}}-1}{\lambda_{4}}\right)$ & $\frac{1}{1-\alpha} \log \left\{\frac{\left(\lambda_{3}-1\right) u^{\lambda_{3}-1}}{\lambda_{3}\left(n \alpha+\lambda_{3}-1\right)}+\frac{\beta_{u}\left(n \alpha+1, \lambda_{4}-2\right)}{u^{n \alpha}}\right\}$ \\
Skew lambda & $\alpha u^{\lambda}-(1-u)^{\lambda}$ & $\frac{1}{1-\alpha} \log \left\{\frac{\alpha \lambda u^{\lambda}}{n \alpha+\lambda}+\frac{\lambda \beta_{u}(n \alpha+1, \lambda)}{u^{n \alpha}}\right\}$ \\
\hline
\end{tabular}

In the following theorem we show that the power distribution can be characterize in terms of $\breve{\xi}_{X_{n: n}}^{\alpha}(u)$.

Theorem 13

Let $X_{n: n}$ denotes the last order statistic with survival function $\bar{F}_{n: n}(x)$ and reverse hazard quantile function 
$\bar{K}_{X_{n: n}}(u)$, then $\breve{\bar{\xi}}_{X_{n: n}}^{\alpha}(u)$ is expressed as

$$
\breve{\bar{\xi}}_{X_{n: n}}^{\alpha}(u)=\frac{1}{(1-\alpha)} \log c-\frac{1}{(1-\alpha)} \log \bar{K}_{X_{n: n}}(u),
$$

if and only if $X$ has power distribution function.

Proof

The reverse hazard quantile function for sample maxima $X_{n: n}$ of power distribution is $\bar{K}_{X_{n: n}}(u)=\frac{f_{n: n}(Q(u))}{F_{n: n}(Q(u))}=$ $\frac{n f(Q(u))}{F(Q(u))}=n(u q(u))^{-1}=\frac{n b u \frac{-1}{b}}{a}$. Taking $c=\frac{n b}{n b \alpha+1}$ gives the if part of the theorem. To prove the only if part, consider (57) be valid. Using (45), it gives

$$
\frac{\int_{0}^{u} p^{n \alpha} q(p) d p}{u^{n \alpha}}=\frac{c}{\bar{K}_{X_{n: n}}(u)} .
$$

Substituting $\bar{K}_{X_{n: n}}(u)=\frac{n}{u q(u)}$, gives

$$
n \int_{0}^{u} p^{n \alpha} q(p) d p=c u^{n \alpha+1} q(u) .
$$

Differentiating both side with respect to $u$ and simplifying, this reduces to

$$
\frac{q^{\prime}(u)}{q(u)}=\left(\frac{n-c(n \alpha+1)}{c}\right) \frac{1}{u} .
$$

Which lead to

$$
q(u)=A u^{\frac{n}{c}-(n \alpha+1)},
$$

where $\mathrm{A}$ is a constant. Which characterizes the power distribution for $c=\frac{n b}{n b \alpha+1}$.

Also we have this characterization in terms of $\bar{M}_{X_{n: n}}(u)$. The proof follows on the same line as Theorem (4.6), hence omitted.

Theorem 14

Let $X_{n: n}$ denotes the last order statistic with survival function $\bar{F}_{n: n}(x)$ and quantile version of mean inactivity time for sample maxima $\bar{M}_{X_{n: n}}(u)$ then $\breve{\xi}_{X_{n: n}}^{\alpha}(u)$ is expressed as

$$
\breve{\xi}_{X_{n: n}}^{\alpha}(u)=\frac{1}{(1-\alpha)} \log c+\frac{1}{(1-\alpha)} \log \bar{M}_{X_{n: n}}(u),
$$

If and only if $X$ has power distribution function.

Remark 2

If $c=\frac{n+1}{n \alpha+1}$, then equation (57) is a characterization of uniform distribution.

\section{Weighted Rényi Quantile Entropy}

Sometimes in statistical modeling, standard distributions are not suitable for our data and we need to study weighted distributions. This concept has been applied in many areas of statistics, such as analysis of family size, human heredity, world life population study, renewal theory, biomedical and statistical ecology. Associated to a random variable $X$ with pdf $f(x)$ and to a nonnegative real function $w(x)$, we can define the weighted random variable $X^{w}$ with density function $f^{w}(x)=\frac{w(x) f(x)}{E(w(X))}, \quad 0<E(w(X))<\infty$. When $w(x)=x, X^{w}$ is called length (size) biased 
random variable. Using $f^{w}(x)$, the corresponding density quantile function is given by

$$
f^{w}(Q(u))=\frac{w(Q(u)) f(Q(u))}{\mu},
$$

where $\mu=\int_{0}^{1} w(Q(p)) f(Q(p)) d(Q(p))=\int_{0}^{1} w(Q(p)) d p$. Weighted entropy has been used to balance the amount of information and degree of homogeneity associated with a partition of data in classes. The quantile-based weighted Rényi entropy is of the form

$$
\breve{\xi}_{w}^{\alpha}(Q(u))=\frac{1}{(1-\alpha)} \log \left(\frac{1}{\mu^{\alpha}} \int_{0}^{1}[w(Q(p))]^{\alpha}(q(p))^{1-\alpha} d p\right) .
$$

In case of length (size) biased random variable the above expression known as length biased weighted Rényi quantile entropy, which is given as

$$
\breve{\xi}_{L}^{\alpha}(Q(u))=\frac{1}{(1-\alpha)} \log \left(\frac{1}{\mu^{\alpha}} \int_{0}^{1}(Q(p))^{\alpha}(q(p))^{1-\alpha} d p\right) .
$$

For some specific univariate continuous distributions, the expression (59) is evaluated as given below in Table 5.1.

Table 5.1 Length biased weighted Rényi quantile entropy $\breve{\xi}_{L}^{\alpha}(Q(u))$ for some lifetime distributions

\begin{tabular}{|c|c|c|}
\hline Distribution & Quantile function $Q(u)$ & $\breve{\xi}_{L}^{\alpha}(Q(u))$ \\
\hline Uniform & $a+(b-a) u$ & $\frac{1}{1-\alpha} \log \left\{\frac{\left(b^{1+\alpha}-a^{1+\alpha}\right) 2^{\alpha}}{(a+b) \alpha^{\alpha}(b-a)^{\alpha}(1+\alpha)}\right\}$ \\
Exponential & $-\lambda^{-1} \log (1-u)$ & $\frac{1}{1-\alpha} \log \left\{\frac{\lambda^{\alpha-1} \gamma(1+\alpha)}{\alpha^{1+\alpha}}\right\}$ \\
Power & $a u^{\frac{1}{b}}$ & $\frac{1}{1-\alpha} \log \left\{\frac{a^{1-\alpha}(b+1)^{\alpha}}{b \alpha+1}\right\}$ \\
Pareto I & $b(1-u)^{-\frac{1}{a}}$ & $\frac{1}{1-\alpha} \log \left\{\frac{b^{1-\alpha}(a-1)^{\alpha}}{(\alpha a-1)}\right\}$ \\
Finite Range & $b\left\{(1-u)^{\frac{1}{a}}\right\}$ & $\frac{1}{1-\alpha} \log \left\{\frac{(a+1)^{\alpha} \beta[1+\alpha, \alpha(a-1)+1]}{a^{-\alpha} b^{\alpha-1}}\right\}$ \\
Log logestic & $\frac{1}{a}\left(\frac{u}{1-u}\right)^{\frac{1}{b}}$ & $\frac{1}{1-\alpha} \log \left\{\frac{a^{\alpha} \beta\left(\alpha+\frac{1}{b}, \alpha-\frac{1}{b}\right)}{a^{2 \alpha} b^{\alpha}\left(\beta\left(1+\frac{1}{b}, 1-\frac{1}{b}\right)\right)^{\alpha}}\right\}$ \\
Weibull & $\left\{-\frac{1}{a} \log (1-u)\right\}^{\frac{1}{b}}$ & $\frac{1}{1-\alpha} \log \left\{\frac{(a b)^{\alpha-1}\left(\frac{1}{a}\right)^{(1-\alpha)\left(\frac{1}{b}-1\right)} \gamma\left(\alpha+\frac{1}{b}\right)}{\alpha^{\alpha+\frac{1}{b}}\left(\gamma\left(1+\frac{1}{b}\right)\right)^{\alpha}}\right\}$ \\
Folded Cramer & $\frac{u}{\theta(1-u)}$ & $\frac{1}{1-\alpha} \log \left\{-\theta^{1-\alpha} \beta(\alpha+1, \alpha-1)\right\}$ \\
Generalized Pareto & $\frac{b}{a}\left\{(1-u)^{-\frac{a}{a+1}}-1\right\}$ & $\frac{1}{1-\alpha} \log \left\{\frac{(-1)^{1+\alpha}\left(\frac{b}{a+1}\right)^{1-\alpha}\left(\frac{a}{a+1}\right) \beta\left[1+\alpha,(1-\alpha)\left(1+\frac{1}{a}\right)\left(\frac{2 a+1}{a+1}\right)-\frac{1}{a}-1\right]}{a^{\alpha}}\right\}$ \\
Pareto II & $\left.a\{1-u)^{-\frac{1}{b}-1}\right\}$ & $\frac{1}{1-\alpha} \log \left\{\frac{b^{\alpha}(b-1)^{\alpha}(-1)^{\alpha+1} \beta[\alpha+1,1-\alpha(b+1)]}{a^{\alpha-1}}\right\}$ \\
Rayleigh & $\left\{-\frac{1}{a} \log (1-u)^{\frac{1}{2}}\right.$ & $\frac{1}{1-\alpha} \log \left\{\frac{2^{\alpha}\left(\frac{1}{\alpha}\right)^{\frac{1}{2}(\alpha-1)} \gamma\left(\alpha+\frac{1}{2}\right)}{\pi^{\frac{\alpha}{2}} \alpha^{\alpha+\frac{1}{2}}(2 a)^{1-\alpha}}\right\}$ \\
\hline
\end{tabular}

Consider a random variable $Y$ with density function $f_{Y}(x)=\frac{\bar{F}(x)}{\mu}$, with $\mu=E(X)<\infty$. Then, ?Y is called the equilibrium random variable of the original random variable $X$, and its distribution as equilibrium distribution of original random variable. The equilibrium distribution arises as the limiting distribution of the forward recurrence time in a renewal process. We have $f_{Y}(Q(u))=\frac{\bar{F}(Q(u))}{\mu}=\frac{1-u}{\mu}$. Thus quantile density function for equilibrium distribution is given by $q_{Y}(u)=\frac{1}{f_{Y}(Q(u))}=\frac{\mu}{1-u}$. From (17), the dynamic cumulative residual Rényi quantile entropy (DCRRQE) for equilibrium distribution is given by

$$
\breve{\xi}^{\alpha}(Y ; Q(u))=\frac{1}{(1-\alpha)} \log \left(\frac{1}{(1-u)^{\alpha}} \int_{u}^{1}(1-p)^{\alpha} q_{Y}(p) d p\right) .
$$


Theorem 15

Let $X$ be a nonnegative random variable with $\breve{\xi}^{\alpha}(Y ; Q(u))=\frac{1}{(1-\alpha)} \log \left(\frac{\mu}{\alpha}\right)$ if and only if $X$ follows equilibrium distribution.

Proof

If part of the theorem is easy to prove, to prove only if part let us assume that $\breve{\xi}^{\alpha}(Y ; Q(u))=\frac{1}{(1-\alpha)} \log \left(\frac{\mu}{\alpha}\right)$. From equation (60), we have

$$
\int_{u}^{1}(1-p)^{\alpha} q_{Y}(p) d p=\frac{\mu(1-u)^{\alpha}}{\alpha}
$$

Differentiating it with respect to $u$ both sides, after some simplification we get $q_{Y}=\frac{\mu}{(1-u)}$, the quantile density function for equilibrium distribution. Hence proved.

Remark 3

The mean residual quantile function satisfy the relation $M(Y ; Q(u))=\mu$ if and only if $X$ follow equilibrium distribution.

\subsection{Weighted Cumulative Residual Rényi Entropy}

Misagh et al. (2011) proposed a weighted information which is based on the $C R E$, called weighted cumulative residual entropy (WCRE). This measure is defined as

$$
\overline{\xi_{w}}(X)=-\int_{0}^{\infty} x \bar{F}(x) \log \bar{F}(x) d x .
$$

Several authors studied properties of (61) and its dynamic version, refer to Kayal and Moharana (2017) and Mirali et al. (2017). As pointed out by Misagh et al. (2011), in some practical situations of reliability and neurobiology a shift-dependent measure of uncertainty is desirable. Also, an important feature of the human visual system is that it can recognize objects in a scale and translation invariant manner. However, achieving this desirable behavior using biologically realistic network is a challenge. The notion of weighted entropy addresses this requirement. In, analogy to (61), The weighted cumulative residual Rényi entropy (WCRRE), and its residual form, defined as

$$
H_{w}^{\alpha}(X)=\frac{1}{1-\alpha} \log \left(\int_{0}^{\infty} x \bar{F}^{\alpha}(x) d x\right), \alpha \neq 0, \alpha>1,
$$

and

$$
H_{w}^{\alpha}(X, t)=\frac{1}{1-\alpha} \log \left(\frac{\int_{t}^{\infty} x \bar{F}^{\alpha}(x) d x}{\bar{F}^{\alpha}(t)}\right),
$$

respectively. The factor $x$ in the integral on right-hand side yields a "length-biased " shift dependent information measure assigning greater importance to larger values of the random variable $X$. From (1) and (63), we propose the quantile version of WCRRE and its residual form of a nonnegative random variable $X$, defined as

$$
\breve{\xi}_{w}^{\alpha}=\frac{1}{(1-\alpha)} \log \left(\int_{0}^{1} Q(u)(1-u)^{\alpha} q(u) d u\right)
$$

and

$$
\breve{\xi}_{w}^{\alpha}(u)=\breve{\xi}_{w}^{\alpha}(X ; Q(u))=\frac{1}{1-\alpha} \log \left\{\frac{\int_{u}^{1} Q(p)(1-p)^{\alpha} q(p) d p}{(1-u)^{\alpha}}\right\},
$$

respectively. The measure (65) may be considered as the dynamic weighted cumulative residual Rényi quantile entropy (DWCRRQE) measure. An alternative expression for the DWCRRQE in terms of mean residual quantile 
function $M(u)$ of random variable $X$ is the following

$$
\breve{\xi}_{w}^{\alpha}(u)=\frac{1}{1-\alpha} \log \left\{\frac{\int_{u}^{1}(1-p)^{\alpha-1} Q(p) M(p) d p}{(1-u)^{\alpha}}-\frac{\int_{u}^{1}(1-p)^{\alpha} Q(p) M^{\prime}(p) d p}{(1-u)^{\alpha}}\right\} .
$$

For some well-known univariate continuous families of distributions, the expression (65) is evaluated as given below in Table 5.2.

Table 5.2 DWCRRQE for several well-known families of distributions

\begin{tabular}{|c|c|c|}
\hline Distribution & Quantile function $Q(u)$ & $\breve{\xi}_{w}^{\alpha}(u)$ \\
\hline Uniform & $a+(b-a) u$ & $\frac{1}{(1-\alpha)} \log \left\{\frac{a(b-a)(1-u)}{1+\alpha}+\frac{(b-a)^{2}(1-u)}{1+\alpha}-\frac{(b-a)^{2}(1-u)^{2}}{2+\alpha}\right\}$ \\
\hline Exponential & $-\lambda^{-1} \log (1-u)$ & $\frac{1}{\gamma(1-\alpha)} \log \left\{\frac{\bar{\gamma}_{-\log (1-u)}(2, \alpha)}{\alpha^{2}(1-\mu)^{\alpha}}\right\}$ \\
\hline Power & $a u^{\frac{1}{b}}$ & $\frac{1}{(1-\alpha)} \log \left\{\frac{a^{2} \bar{\beta}_{u}\left(\frac{2}{b}, 1+\alpha\right)}{b(1-u)^{\alpha}}\right\}$ \\
\hline Pareto I & $b(1-u)^{-\frac{1}{a}}$ & $\frac{1}{1-\alpha} \log \left\{\frac{b^{2}(1-u)^{\frac{-2}{a}}}{(\alpha a-2)}\right\}$ \\
\hline Folded Cramer & $\frac{u}{\theta(1-u)}$ & $\frac{1}{(1-\alpha)} \log \left\{\frac{\bar{\beta}_{u}(2, \alpha-2)}{\theta^{2}(1-u)^{\alpha}}\right\}$ \\
\hline Generalized Pareto & $\frac{b}{a}\left[(1-u)^{-\frac{a}{a+1}}-1\right]$ & $\frac{1}{(1-\alpha)} \log \left\{\frac{b^{2}(1-u)^{\frac{-2 a}{a+1}}}{a(a \alpha+\alpha-2 a)}-\frac{b^{2}(1-u)^{\frac{-a}{a+1}}}{a(\alpha a+\alpha-a)}\right\}$ \\
\hline Finite Range & $b\left\{1-(1-u)^{\frac{1}{a}}\right\}$ & $\frac{1}{(1-\alpha)} \log \left\{\frac{b^{2}(1-u)^{\frac{1}{a}}}{(a \alpha+1)}-\frac{b^{2}(1-u)^{\frac{2}{a}}}{(\alpha a+2)}\right\}$ \\
\hline Log logestic & $\frac{1}{a}\left(\frac{u}{(1-u)}\right)^{\frac{1}{b}}$ & $\frac{1}{(1-\alpha)} \log \left\{\frac{\bar{\beta}_{u}\left(\frac{2}{b}, \alpha-\frac{2}{b}\right)}{a^{2} b(1-u)^{\alpha}}\right\}$ \\
\hline Weibull & $\left\{-\frac{1}{a} \log (1-u)\right\}^{\frac{1}{b}}$ & $\frac{1}{(1-\alpha)} \log \left\{\frac{\left(\frac{1}{a}\right)^{\frac{2}{b}-1} \bar{\gamma}_{-\log (1-u)}\left(\frac{2}{b}, \alpha\right)}{a b(1-u)^{\alpha}}\right\}$ \\
\hline Rayleigh & $\left\{-\frac{1}{a} \log (1-u)\right\}^{\frac{1}{2}}$ & $\frac{1}{(1-\alpha)} \log \left\{\frac{1}{2 a \alpha}\right\}$ \\
\hline Gompertz & $\frac{1}{\log C}\left\{1-\frac{\log C \log (1-u)}{B}\right\}$ & $\frac{1}{(1-\alpha)} \log \left\{\frac{1}{\alpha B \log C}+\frac{\bar{\gamma}_{-\log (1-u)}(2, \alpha)}{B^{2}(1-u)^{\alpha}}\right\}$ \\
\hline Govindarajulu's & $a\left\{(b+1) u^{b}-b u^{b+1}\right\}$ & $\frac{1}{(1-\alpha)} \log \left\{\frac{a^{2} b(b+1)^{2} \bar{\beta}_{u}(2 b, \alpha+2)}{(1-u)^{\alpha}}-\frac{a^{2} b^{2}(b+1) \bar{\beta}_{u}(2 b+1, \alpha+2)}{(1-u)^{\alpha}}\right\}$ \\
\hline
\end{tabular}

\section{Example 8}

Let $X$ follows lambda family of distribution as given in example (15), then weighted cumulative residual Rényi quantile entropy $(W C R R E)(64)$ is given as

$$
\breve{\xi}_{w}^{\alpha}=\frac{1}{(1-\alpha)} \log \left\{C^{2} \lambda_{1} \beta\left(2 \lambda_{1}, 1+\alpha\right)+C^{2} \lambda_{2} \beta\left(2 \lambda_{1}+1, \alpha-2 \lambda_{2}\right)\right\} .
$$

As $\lambda_{1} \longrightarrow 0$, (66) reduces to $\breve{\xi}_{w}^{\alpha}=\frac{1}{(1-\alpha)} \log \left(\frac{C^{2} \lambda_{2}}{\alpha-2 \lambda_{2}}\right)$, corresponding to Pareto I distribution. Also, as $\lambda_{2} \longrightarrow 0$, (66) reduces to $\breve{\xi}_{w}^{\alpha}=\frac{1}{(1-\alpha)} \log \left(C^{2} \lambda_{1} \beta\left(2 \lambda_{1}, 1+\alpha\right)\right)$, corresponding to power distribution.

In order to provide some characterization results for $D W C R R Q E$ of a nonnegative random variable $X$. Let us define the quantile version of weighted mean residual lifetime (WMRE), as follows

$$
M^{w}(u)=m^{w}(Q(u))=\frac{\int_{u}^{1}(1-p) Q(p) q(p) d p}{1-u},
$$

here $m^{w}(t)=\frac{\int_{t}^{\infty} x \bar{F}(x) d x}{\bar{F}(t)}$ is the WMRE of random variable $X$. In the following theorem, we characterize rayleigh distribution using a relationship between DWCRRQE and quantile-basedWMRE. 
Theorem 16

Let $X$ be an absolutely continuous random variable. Then the relation

$$
\breve{\xi}_{w}^{\alpha}(X ; Q(u))=\frac{1}{(1-\alpha)} \log \left(\frac{M^{w}(u)}{\alpha}\right),
$$

holds if and only if $X$ follows the rayleigh distribution.

Proof

The quantile-basedWMRE (67), for rayleigh distribution is given as

$$
M^{w}(u)=\frac{1}{2 a(1-u)} \int_{u}^{1}\left(\frac{-1}{a} \log (1-p)\right)^{\frac{1}{2}}\left(\frac{-1}{a} \log (1-p)\right)^{\frac{-1}{2}} d p=\frac{1}{2 a} .
$$

The weighted cumulative residual Rényi quantile entropy (65), for rayleigh distribution is

$$
\breve{\xi}_{w}^{\alpha}(X ; Q(u))=\frac{1}{1-\alpha} \log \left(\frac{1}{2 a \alpha}\right) .
$$

This prove the if part of the Theorem. To prove only if part, let (68) holds. Then

$$
\alpha \int_{u}^{1}(1-p)^{\alpha} Q(p) q(p) d p=(1-u)^{\alpha-1} \int_{u}^{1}(1-p) Q(p) q(p) d p .
$$

Differentiating both sides with respect to $u$, we have

$$
(1-u)^{\alpha} q(u) Q(u)=(1-u)^{\alpha-2} \int_{u}^{1}(1-p) Q(p) q(p) d p .
$$

Using (67), we have

$$
Q(u) q(u)=\frac{M^{w}(u)}{1-u} .
$$

Differentiating (67) with respect to $u$ both sides, we have

$$
\frac{d M^{w}(u)}{d u}-\frac{M^{w}(u)}{1-u}=-Q(u) q(u)
$$

Substituting in (69), gives $\frac{d M^{w}(u)}{d u}=0$ or equivalently $M^{w}(u)=k$ (constant), which characterizes the rayleigh distribution.

\section{Theorem 17}

For a nonnegative random variable $X$, the relationship

$$
\breve{\xi}_{w}^{\alpha}(X ; Q(u))=C,
$$

where $C$ is a constant holds, then $X$ has the rayleigh distribution.

Proof

The necessary part follows from the Table (60). For the sufficiency part, let us assume (70) holds. From (65) we have

$$
e^{(1-\alpha) \breve{\xi}_{w}^{\alpha}(X ; Q(u))}(1-u)^{\alpha}=\int_{u}^{1} Q(p)(1-p)^{\alpha} q(p) d p .
$$

Taking derivative with respect to $\mathrm{u}$ both sides we have, after some algebraic simplification

$$
\left((1-\alpha) \breve{\xi}_{w}^{\prime} \underset{w}{\alpha}(X ; Q(u))-\frac{\alpha}{(1-u)}\right) e^{(1-\alpha) \breve{\xi}_{w}^{\alpha}(X ; Q(u))}=-q(u) Q(u)
$$


Using (4), this gives

$$
\left((1-\alpha) \breve{\xi}_{w}^{\prime \alpha}(X ; Q(u))-\frac{\alpha}{(1-u)}\right) e^{(1-\alpha) \breve{\xi}_{w}^{\alpha}(X ; Q(u))}=-\frac{Q(u)}{(1-u) K(u)} .
$$

From (70), we get $\breve{\xi}_{w}^{\prime}(X ; Q(u))=0$. Substitute this value in the above expression we obtain

$$
\alpha e^{(1-\alpha) \breve{\xi}_{w}^{\alpha}(X ; Q(u))} K(u)-Q(u)=0,
$$

which leads to $\frac{K(u)}{Q(u)}=\frac{1}{\alpha e^{C(1-\alpha)}}=2 a$ (constant). Thus $X$ follows rayleigh distribution with survival function $\bar{F}(x)=\exp \left(-\frac{x}{2 a^{2}}\right)$. Hence, the proof is completed.

\section{Definition 1}

The distribution function $F$ is said to be increasing (decreasing) in dynamic weighted cumulative residual Rényi quantile entropy IDWCRRQE (DDWCRRQE) if $\breve{\xi}_{w}^{\alpha}(X ; Q(u))$ is increasing (decreasing) in $u \geq 0$.

The following theorem gives the upper (lower) bound to the DWCRRQE, in terms of the hazard quantile function.

Theorem 18

The distribution function $F$ is IDWCRRQE (DDWCRRQE), if and only if, for all $u \geq 0$.

$$
\begin{gathered}
\breve{\xi}_{w}^{\alpha}(X ; Q(u)) \geq(\leq) \frac{1}{(1-\alpha)} \log \left(\frac{Q(u)}{\alpha K(u)}\right), \quad \alpha>1, \\
\breve{\xi}_{w}^{\alpha}(X ; Q(u)) \leq(\geq) \frac{1}{(1-\alpha)} \log \left(\frac{Q(u)}{\alpha K(u)}\right), \quad 0 \leq \alpha<1 .
\end{gathered}
$$

\section{Conclusion}

Quantile-based study of entropy measures found greater interest among researchers as an alternative method of measuring uncertainty of random variable. In this paper we have proposed dynamic cumulative residual Rényi quantile entropy and studied some properties, characterizations. We have introduced the quantile-based cumulative residual Rényi entropy of order statistics and its characterizations. We have also obtained the weighted Rényi quantile entropy and its residual form based on cumulative function and obtain some characteristic result.

\section{Acknowledgement}

The authors would like to express their gratitude to the reviewers and the editor in chief for their valuable comments, which have considerably improved the earlier version of the article. The corresponding author wishes to acknowledge the Science and Engineering Research Board (SERB) New Delhi, Government of India, for the financial assistance (Ref. No. ECR/2017/001987) for carrying out this research work.

\section{REFERENCES}

1. B. C. Arnold, N. Balakrishnan, and H. N. Nagaraja, A First Course in Order Statistics, John Wiley and Sons, New York, 1992.

2. M. Asadi, and Y. Zohrevand, On the dynamic cumulative residual entropy, Journal of Statistical Planning and Inference, vol. 137, pp. 1931-1941, 2007.

3. H. A. David, and H. N. Nagaraja, Order Statistics, John Wiley and Sons, New York, 2003.

4. A. Di Crescenzo, and M. Longobardi, On cumulative entropies, Journal of Statistical Planning and Inference, vol. 139, no. 12, pp. 4072-4087, 2009.

5. M.Fashandi, and J. Ahmadi, Characterizations of symmetric distributions based on Rényi entropy, Statistics and Probability Letters, vol. 82, no. 1, pp. 798-804, 2012. 
6. Z. Govindarajulu, A class of distributions useful in lifetesting and reliability with applications to nonparametric testing, In: C.P. Tsokos, I.N. Shimi (eds.) Theory and Applications of Reliability, Acad. Press, New York., vol. 1, pp. 109-130, 1977.

7. R. K. S. Hankin, and A. Lee, A new family of nonnegative distributions, Australian and New Zealand Journal of Statistics, vol. 48, pp. 67-78, 2006.

8. S. Kayal, and R. Moharana, On weighted cumulative residual entropy, Journal of Statistics and Management Systems, vol. 20, pp. 153-173, 2017.

9. S. Kayal, On generalized dynamic survival and failure entropies of order $(\alpha, \beta)$, . Statistics and Probability Letters, vol. 96 , no. 2 pp. $123-132,2015$.

10. M. Mirali, S. Baratpour, and V. Fakoor, On weighted cumulative residual entropy, Communications in Statistics-Theory and Methods, vol. 46, pp. 2857-2869, 2017.

11. F. Misagh, Y. Panahi, G.H. Yari, and R. Shahi, Weighted Cumulative entropy and its estimation, IEEE International Conference on Quality and Reliability (ICQR), 12287011, 2011.

12. N. U. Nair, and P. G. Sankaran, Quantile-based reliability analysis, Communications in Statistics Theory and Methods, vol. 38, no 2 pp. $222-232,2009$.

13. N. U. Nair, and P. G. Sankaran, and N. Balakrishnan, Quantile-based reliability analysis, Springer-Verlag, New York, 2013.

14. N. U. Nair, and P. G. Sankaran, and B. Vinesh Kumar Modeling lifetimes by quantile functions using Parzens score function, Statistics, vol. 46, pp. 799-811, 2011.

15. J. Navarro, Y. del Aguila, and M. Asadi, Some new results on the cumulative residual entropy, Journal of Statistical Planning and Inference, vol. 140, no. 1, pp. 310-322, 2010.

16. E. Parzen, Non parametric statistical data modeling, Journal of American Statistical Association, vol. 74, pp. $105-122,1979$.

17. M. Rao, Y. Chen, B. C. Vemuri, and F. Wang, Cumulative residual entropy: a new measure of information IEEE Transactions on Information Theory, vol. 50, pp. 1220-1228, 2004.

18. M. Rao, More on a new concept of entropy and information, Journal of Theoretical Probability, vol. 18, pp. 967-981, 2005.

19. P. G. Sankaran, and S. M. Sunoj, Quantile-based cumulative entropies, Communications in Statistics Theory and Methods, vol. 46, no. 2, pp. 805-814, 2017.

20. V. Kumar, and N. Singh Characterization of Lifetime Distribution Based on Generalized Interval Entropy, Statistics, Optimization and Information Computing, vol. 6, no. 4, pp. 547-559 2018.

21. C. E. Shannon, A mathematical theory of communication., Bell System Technical Journal, vol. 27, pp. 379-423, 1948.

22. N. Sreelakshmi, S. K. Kattumannil, and G. Asha, Quantile-based tests for expontiality against dmrq and nbue alternatives, Journal of Korean Statistics Society, vol. 47, no. 2, pp. 185-200, 2018.

23. S. M. Sunoj, A. S. Krishnan, and P. G. Sankaran, Quantile-based entropy of order statistics, Journal of the Indian Society for Probability and Statistics, vol. 18, pp. 1-17, 2017

24. S. M. Sunoj, and M. N. Linu, Dynamic cumulative residual Rényi entropy, A journal of Theoretical and Applied Statistics, vol. 46, no. 1, pp. 41-56, 2012.

25. S. M. Sunoj, and P. G. Sankaran, Quantile-based entropy function, Statistics and Probability Letters, vol. 82, pp. 1049-1053, 2012.

26. S. M. Sunoj, and P. G. Sankaran, and A. K. Nanda, Quantile-based entropy function in past lifetime, Statistics and Probability Letters, vol. 83, pp. 366-372, 2013.

27. R. Thapliyal, and H. C. Taneja, On Rényi entropies of order statistics, International Journal of Biomathematics, vol. 8, no. 6, 1550080,2015

28. F. Wang, and B. C. Vemuri, Non-Rigid multimodal image registration using cross-cumulative residual entropy, International Journal of Computer Vision, vol. 74, no. 2, pp. 201-215, 2007.

29. P. J. Van Staden, and M. R. Loots, L-moment estimation for the generalized lambda distribution, ed., Third Annual ASEARC Conference, New Castle, Australia, 2009.

30. K. Zografos, and S. Nadarajah, Survival exponential entropies, IEEE Transation Information Theory, vol. 51, pp. 1239-1246, 2005.

31. S. Minimol, On generalized dynamic cumulative past entropy measure, Communications in Statistics-Theory and Methods, vol.46, no. 6 , pp. $2816 ? 2822,2017$.

32. S. Baratpour, A. H. Khammar, A quantile-based generalized dynamic cumulative measure of entropy, Communications in StatisticsTheory and Methods, vol. 47, no. 13, pp. 3104-3117, 2018.

33. M. Sheraz, S. Dedub, V. Predaa, Entropy Measures for Assessing Volatile Markets, Procedia Economics and Finance, vol. 22, pp. $655-662,2015$.

34. W. Gilchrist, Statistical modelling with quantile functions, Chapman and Hall/CRC, Boca Raton, FL, 2000.

35. M. Abbasnejad, N. R. Arghami, Rényi entropy properties of order statistics, Communications in Statistics-Theory and Methods, vol. 40, pp. 40-52, 2011

36. V. Kumar, and N. Singh Quantile-based Generalized Entropy of order $(\alpha, \beta)$ for Order Statistics, STATISTICA, vol. 78, no. 4, pp. 299-318, 2019.

37. R. C. Gupta, H. C. Taneja, and R. Thapliyal, Stochastic comparisons of residual entropy of order statistics and some characterization results, Journal of Statistical Theory and Applications, vol. 13, no. 1, pp. 27-37, 2014.

38. D. Sharma, and T. K. Chakrabarty, Some General Results On Quantile Functions For The Generalized Beta Family, Statistics, Optimization and Information Computing, Vol.5, pp. 360?377, 2017.

39. H. A. Noughabi, H. A. Noughabi, J. Jarrahiferiz. Informational Energy and Entropy Applied to Testing Exponentiality, Statistics, Optimization and Information Computing, Vol.8, no.1, pp. 220?228, 2020. 